Appl Microbiol Biotechnol (1990) 32:393-397

\title{
Effects of extractive fermentation on butyric acid production by Clostridium acetobutylicum
}

\author{
Patrick J. Evans* and Henry Y. Wang \\ Department of Chemical Engineering, The University of Michigan, Ann Arbor, MI 48109, USA
}

Summary. The addition of an oleyl alcohol extractant to a batch fermentation of glucose by Clostridium acetobutylicum resulted in a concentration profile that was distinctly different from the non-extractive control fermentation. The concentration of butyric acid increased and subsequently decreased in the control fermentation. The concentration of butyric acid increased but did not subsequently decrease in the oleyl alcohol extractive fermentation. The production of butyric acid was found to have been prolonged into the solventogenic phase in the oleyl alcohol extractive fermentation. Butyric acid was continually replenished from glucose while it was being converted to butanol. Supplementation of exogenous acetic and butyric acids, the metabolic uncoupler carbonyl cyanide 3-chlorophenylhydrazone, or decanol to the oleyl alcohol extractive fermentation helped to reinstate the normal butyric acid concentration profile. These findings are discussed with respect to the effects of these additives on the $\Delta \mathrm{pH}$ of $C$. acetobutylicum and its importance with regard to the production of butyric acid.

\section{Introduction}

Batch fermentation of glucose by Clostridium acetobutylicum is characterized by the initial production of acetic and butyric acids (acidogenesis) followed by the conversion of these acids to ethanol and butanol and the production of acetone (solventogenesis). Butyric acid has been shown to have a role in triggering the onset of solventogen-

* Present address: Department of Microbiology, NYU Medical Center, 550 First Ave., New York, NY 10016, USA

Offprint requests to: $\mathrm{H}$. Y. Wang esis and the associated conversion of acids to solvents in C. acetobutylicum (Bahl et al. 1982; Ballongue et al. 1985; Fond et al. 1985; Gottshal and Morris 1981; Gottwald and Gottschalk 1985; Holt et al. 1984; Huang et al. 1985; Huang et al. 1986; Huesemann and Papoutsakis 1986; Terracciano and Kashket 1986); however, the molecular mechanism is unknown.

Organic solvents can be used to enhance the production of butanol in extractive fermentation. The extraction of butyric acid from broth during extractive fermentation has not been observed by other investigators (Roffler et al. 1987; Taya et al. 1985; Y. J. Jeon and Y. Y. Lee presentation at the Eighth Symposium on Biotechnology for Fuels and Chemicals, Gatlinburg, Tenn, 1986). However, in our system, butyric acid was extracted from the fermentation broth into oleyl alcohol (Evans and Wang 1988). This extraction lowered the aqueous butyric acid concentration relative to the butyric acid concentration in the fermentation without extraction.

Extractive fermentation of $C$. acetobutylicum with an equal volume of oleyl alcohol caused an increase in the final yields of acetic and butyric acids (Evans and Wang 1988). On the other hand, the use of an extractant composed of $30 \%$ (vol/ vol) decanol and $70 \%$ (vol/vol) oleyl alcohol resulted in final acid yields that were similar to those observed without any extractant. The purpose of this study was to examine how decanol and oleyl alcohol affected the production of butyric acid during solventogenesis in an extractive fermentation.

\section{Materials and methods}

Microorganism and culture conditions. Clostridium acetobutylicum ATCC 4259 was grown in CAB medium with $60 \mathrm{~g} / \mathrm{I}$ of 
glucose at a $\mathrm{pH}$ of 4.5 as described previously (Evans and Wang 1988) unless otherwise indicated. Equal volumes (300 $\mathrm{ml}$ each) of extractant and broth were used. The extractant was composed of oleyl alcohol (Kodak, Rochester, NY, USA) and decanol (Aldrich, Chicago, IIl, USA). The control fermentation contained no extractant. Where indicated, butyric and acetic acids were added to the fermentor prior to sterilization. Carbonyl cyanide 3-chlorophenylhydrazone (CCCP), a metabolic uncoupler, was added to the culture broth as an aqueous solution of its sodium salt. A volume of $11.8 \mathrm{ml}$ of a $3.2 \mathrm{mM}$ CCCP solution was added which resulted in an aqueous concentration of $24 \mu M$ at the time of inoculation. Most of the CCCP was extracted into the organic phase.

Rate of conversion of butyric acid to butanol. The rate of conversion of butyric acid to butanol was determined by measurement of the rate of production of labelled butanol from labelled butyric acid. At various times, $5 \mathrm{ml}$ whole broth was removed anaerobically from an oleyl alcohol extractive fermentation. The broth was added to an anaerobic pressure tube (Bellco Glass, Vineland, NJ, USA) with $2 \mu \mathrm{Ci}$ of $\left[1-{ }^{14} \mathrm{C}\right]$ butyric acid $(30 \mu M, 13.4 \mu \mathrm{Ci} / \mu \mathrm{mol}$, New England Nuclear, Boston, Mass, USA). The broth samples were incubated at $34^{\circ} \mathrm{C}$ for $15 \mathrm{~min}$ and then two $1 \mathrm{ml}$ samples were removed and centrifuged. Aliquots $(200 \mu \mathrm{l})$ of each supernatant were added to scintillation vials with $20 \mathrm{ml}$ Safety-Solve scintillation cocktail (Research Products International, Mount Prospect, Ill, USA) and counted to determine the initial labelled butyric acid concentration. Aliquots $(500 \mu \mathrm{l})$ of each supernatant were added to $100 \mu l$ of $1 \mathrm{~N} \mathrm{NaOH}$ to dissociate the unconverted butyric acid into the anionic form. Pentanol $(1 \mathrm{ml})$ was added to this solution and vortexed for $5 \mathrm{~s}$ to extract the butanol from the butyric acid. The extracts were centrifuged to separate emulsified water and $200 \mu 1$ of each of the organic phases were added to scintillation vials with $20 \mathrm{ml}$ Safety-Solve and counted. The concentration of labelled butanol in the broth was calculated from the number of counts per minute and a dilution factor. Corrections were made for extracted butyric acid (3\%) and unextracted butanol $(6 \%)$. The rate of butyric acid conversion was calculated from the amount of labelled butanol formed in $15 \mathrm{~min}$, the initial concentration of labelled butyric acid, and the initial concentration of unlabelled butyric acid, which was measured by gas chromatography. The results for the duplicate samples were averaged.

Analytical techniques. Product concentrations were determined in the aqueous and organic phases by gas chromatography as described previously (Evans and Wang 1988). The total concentrations of butyric acid, acetone and butanol were calculated by addition of the organic and aqueous concentrations. Total concentration is based on the aqueous phase volume.

The protein content of the cells was determined by the Biuret method (Herbert et al. 1971) with cell digestion in $3 \mathrm{~N}$ $\mathrm{NaOH}$ at $100^{\circ} \mathrm{C}$ for $5 \mathrm{~min}$. The standard was bovine serum albumin.

The concentration of CCCP was assayed by UV spectroscopy. In citrate-phosphate buffer at a $\mathrm{pH}$ of $4.5, \mathrm{CCCP}$ has absorption maxima at 220 and $355 \mathrm{~nm}$. The assay was done at $355 \mathrm{~nm}$. Against a CAB medium blank, the extinction coefficient of CCCP in CAB medium was $18 \mathrm{mM}^{-1} \mathrm{~cm}^{-1}$.

\section{Results}

The control fermentation had no extractant. The extractive fermentations contained an organic phase volume equal to that of the broth. The first extractant was oleyl alcohol and the second one was a mixture of $30 \%(\mathrm{vol} / \mathrm{vol})$ decanol and $70 \%$ (vol/vol) oleyl alcohol. Figure $1 \mathrm{a}$ and $\mathrm{b}$ shows that the rates of acid production during acidogenesis were similar in the control fermentation and the two extractive fermentations. The acid concentration profile during the solventogenic phase of the oleyl alcohol extractive fermentation differed from the profiles of the other two fermentations. Decreases in the acetic and butyric acid concentrations during solventogenesis were less in magnitude in the oleyl alcohol extractive fermentation than decreases in the other two fermen-
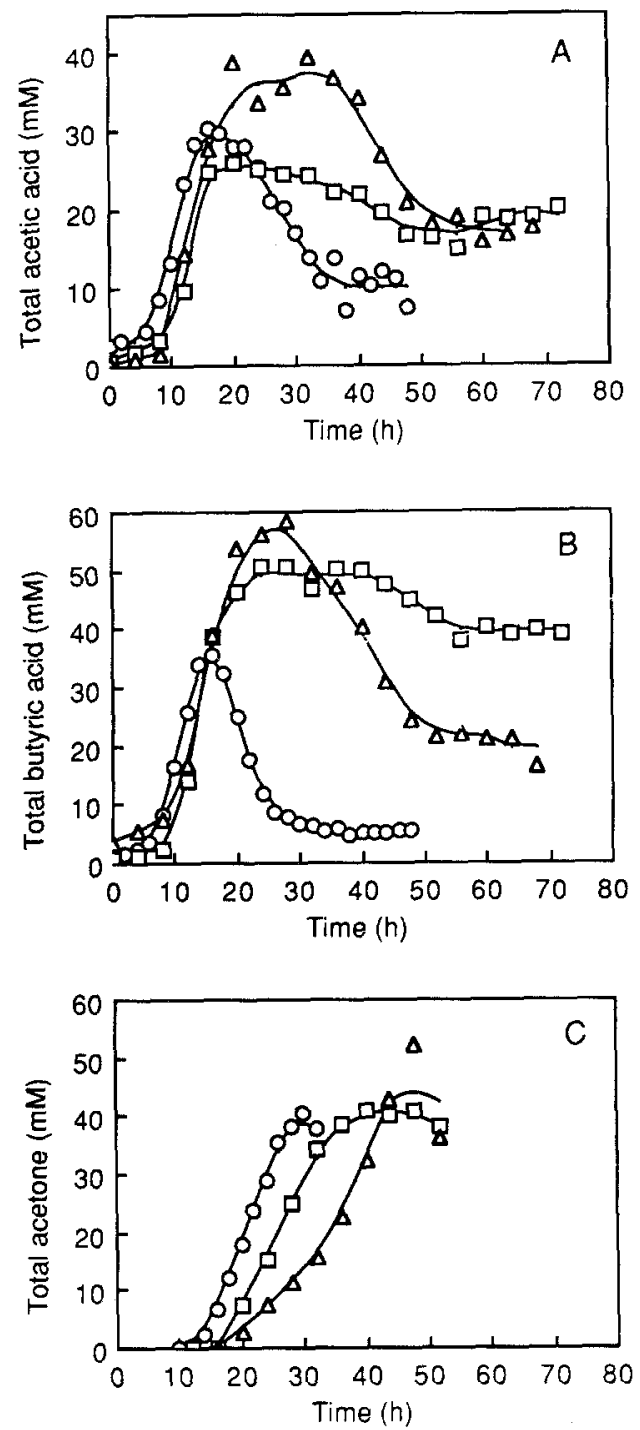

Fig. 1A-C. Concentration profiles for batch fermentations: control without extractant $(O)$, with oleyl alcohol $(\square)$, and with $30 \%$ decanol in oleyl alcohol $(\triangle)$. All concentrations are the total concentrations. A Acetic acid. B Butyric acid. C Acetone 
tations $(0.01<p<0.025$ for butyric acid and $0.01<p<0.1$ for acetic acid, based on duplicate fermentations and a one-sided comparison). The rates at which the acid concentrations decreased during solventogenesis were also less in magnitude in the oleyl alcohol extractive fermentation than in the other two fermentations $(0.005<p<0.05$ for butyric acid and $0.025<p<0.1$ for acetic acid). The control fermentation and the decanol-oleyl alcohol extractive fermentation were not significantly different from each other based upon the same analysis.

Differences in the acid profiles during solventogenesis in the two extractive fermentations were not attributable to differences in the aqueous, butyric acid concentrations. The maximal aqueous concentrations of butyric acid in the control, oleyl alcohol, and decanol-oleyl alcohol fermentations were 36,20 and $21 \mathrm{mM}$, respectively.

The acetone concentration profiles of all three fermentations, on the other hand, were similar. Figure 1c shows no differences in acetone production upon comparison of the oleyl alcohol extractive fermentation with the control fermentation. Based on duplicate fermentations, the volumetric production rates of acetone were $55 \pm 11$, $49 \pm 15$, and $59 \pm 23 \mathrm{nmol} \mathrm{min}{ }^{-1} \mathrm{ml}^{-1}$ in the control, oleyl alcohol, and decanol-oleyl alcohol fermentations, respectively. Thus, no differences were found in the rates of acetone production among the three fermentations.

The oleyl alcohol extractive fermentation again was unique in the relationship of the acid concentration profile to acetone production. Comparison of Fig. 1a and b to Fig. 1c shows that the decrease in butyric acid concentration occurred simultaneously to the increase in acetone concentration in both the control and the decanol-oleyl alcohol extractive fermentation. This did not occur in the oleyl alcohol extractive fermentation. The ratios of the decrease in acid concentration (acetic plus butyric) to the production of acetone $\left(\Delta \mathrm{C}_{\text {acid decrease }} / \Delta \mathrm{C}_{\text {acetone }}\right)$ for the control, oleyl alcohol, and decanol-oleyl alcohol fermentations in Fig. 1 were 1.3, 0.58, and 1.3, respectively. Acetone production may have been underestimated because of its volatility; consequently the above ratios may have been overestimated. Similar results were observed in replicate fermentations.

The specific rates of decrease in acetic and butyric acid concentrations and increase in acetone formation that correspond to the data in Fig. 1 are presented in Table 1. The specific rates of decrease in the acid concentration were less in the oleyl alcohol extractive fermentation than in the
Table 1. Maximal specific rates ( $\mathrm{nmol} \mathrm{min}^{-1} \mathrm{mg}_{\text {protein }}{ }^{-1}$ ) of acid concentration decrease and acetone production

\begin{tabular}{llll}
\hline Fermentation & \multicolumn{2}{l}{$\begin{array}{l}\text { Decrease in } \\
\text { concentration }\end{array}$} & $\begin{array}{l}\text { Acetone } \\
\text { formation }\end{array}$ \\
\cline { 2 - 3 } & Acetic & Butyric & \\
\hline $\begin{array}{l}\text { Control } \\
\begin{array}{l}\text { Oleyl alcohol } \\
\text { Decanol plus } \\
\text { oleyl alcohol }\end{array}\end{array}$ & 11 & 72 & 59 \\
\hline
\end{tabular}

other two fermentations. The specific rate of acetone formation was not less in the oleyl alcohol extractive fermentation than in the other two fermentations.

The concentration of butyric acid was affected by both the production of butyric acid and the conversion of butyric acid to butanol, as illustrated by a differential mole balance on butyric acid:

$d C / d t=r_{\text {prod }}-r_{\text {conv }}$

where $d C / d t$ is the rate of change in the butyric acid concentration, $r_{\text {prod }}$ is the volumetric production rate of butyric acid and $r_{\text {conv }}$ is the volumetric rate of conversion of butyric acid to butanol. Table 2 illustrates that the rate of conversion of butyric acid to butanol in the oleyl alcohol extractive fermentation was correlated with the rate of production of acetone. The maximal rate of acetone production during this fermentation was 38 $\mathrm{nmol} \min ^{-1} \mathrm{ml}^{-1}$ between 15 and $20 \mathrm{~h}$. The maximal butyric acid concentration was observed at $25 \mathrm{~h}$. Calculation of $r_{\text {prod }}$ was by addition of $r_{\text {canv }}$ and $d C / d t$. The data show that the production of butanol was prolonged into the solventogenic phase.

Table 2. Volumetric rates (nmol $\mathrm{min}^{-1} \mathrm{ml}^{-1}$ ) of acetone production, butyric acid conversion to butanol $\left(r_{\text {conv }}\right)$, change in butyric acid concentration $(d C / d t)$, and butyric acid production $\left(r_{\text {prod }}\right)$ in the extractive oleyl alcohol fermentation

\begin{tabular}{lllll}
\hline \multirow{2}{*}{$\begin{array}{l}\text { The } \\
\text { (h) }\end{array}$} & $\begin{array}{l}\text { Acetone } \\
\text { production }\end{array}$ & \multicolumn{3}{l}{ Butyric acid } \\
\cline { 3 - 5 } & & $r_{\text {conv }}$ & $d C / d t$ & $r_{\text {prod }}$ \\
\hline 10 & ND $^{\mathrm{a}}$ & 5.4 & +44 & 49 \\
21 & 26 & 33 & +6.3 & 39 \\
23 & 26 & 32 & +6.3 & 38 \\
35 & 6.6 & 16 & -5.2 & 11 \\
\hline
\end{tabular}

${ }^{a}$ Onset of acetone production 
Table 3. Decrease in acid concentrations during solventogenesis in the oleyl alcohol extractive fermentation upon the addition of acids and carbonyl cyanide 3-chlorophenylhydrazone (CCCP)

\begin{tabular}{|c|c|c|c|c|}
\hline \multirow[t]{2}{*}{ Addition } & \multicolumn{2}{|c|}{$\begin{array}{l}\text { Concentration decrease } \\
(\mathrm{m} M)\end{array}$} & \multicolumn{2}{|c|}{$\begin{array}{l}\text { Rate of decrease } \\
\left(\mathrm{nmol} \mathrm{min} \mathbf{m}^{-1} \mathrm{ml}^{-1}\right)\end{array}$} \\
\hline & Acetic & Butyric & Acetic & Butyric \\
\hline None & $9.5(2.1)^{\mathrm{a}}$ & $13(1)$ & $9.4(3.7)$ & $13(2)$ \\
\hline Acids & ND & $89^{b}$ & ND & $50^{\mathrm{c}}$ \\
\hline $\mathrm{CCCP}$ & $17^{d}$ & $25^{\mathrm{c}}$ & 10 & $18^{\mathrm{d}}$ \\
\hline
\end{tabular}

Numbers in parentheses are $1 \mathrm{SD}$

${ }^{\mathrm{b}} p<0.005$

c $p<0.025$

${ }^{\mathrm{d}} p<0.1$

Since butyric acid was extracted from the broth in the presence of oleyl alcohol, acetic and butyric acids were supplemented to the fermentation broth in the presence of oleyl alcohol. The results are presented in Table 3 . Acetic acid $(30 \mathrm{~m} M)$ and butyric acid $(110 \mathrm{~m} M$ total, $34 \mathrm{mM}$ aqueous) were added to the broth prior to inoculation. The decrease in the total butyric acid concentration during solventogenesis in the oleyl alcohol extractive fermentation was significantly enhanced upon acid addition. The uncoupler CCCP was added to the oleyl alcohol extractive fermentation to test whether a lower $\Delta \mathrm{pH}$ could enhance the decrease in acid concentrations during solventogenesis. The amount of decrease in the total butyric acid concentration during solventogenesis was enhanced upon the addition of CCCP ( $24 \mu M$ aqueous) to the oleyl alcohol extractive fermentation. The rate of this decrease was also enhanced. The amount of decrease in the acetic acid concentration during solventogenesis was enhanced by the presence of CCCP. Greater concentrations of CCCP could not be used because of its toxicity. The enhancement of the decrease in acid concentrations during solventogenesis in the presence of CCCP may have been limited by depletion of glucose. Glucose was not depleted in the fermentations without CCCP. The supplementation of acids and CCCP to the oleyl alcohol extractive fermentation resulted in a lower rate of acetone production relative to the unsupplemented oleyl alcohol extractive fermentation. The volumetric rates of acetone production were $49 \mathrm{nmol} \mathrm{min}{ }^{-1} \mathrm{ml}^{-1}$ with no addition, 30 $\mathrm{nmol} \min ^{-1} \mathrm{ml}^{-1}$ with acid addition, and 36 nmol $\min ^{-1} \mathrm{ml}^{-1}$ with CCCP addition.

\section{Discussion}

The acetic and butyric acid concentration profiles of the oleyl alcohol extractive fermentation (Type I) were found to be different from the profiles of the control and decanol-oleyl alcohol extractive fermentations (Type II). These differences occurred in the solventogenic phase. The amount of decrease and the rate of decrease in acid concentration during solventogenesis was less in the Type I fermentation than in the Type II fermentation (Fig. 1). The difference between the Type I and Type II acid concentration profiles was not attributable to different cell concentrations; rather, it was attributable to physiological differences in the cells (Table 1).

Acetone production was not affected by the addition of oleyl alcohol to the fermentation broth. Thus the acid concentration profiles were affected independently of acetone production. Furthermore, the ratio $\Delta C_{\text {acid decrease }} / \Delta C_{\text {acetone }}$ was less than one only in the oleyl alcohol extractive fermentation. The behavior of the oleyl alcohol extractive fermentation was a paradox since increases in the acetone concentration typically correlate with decreases in the acetic and butyric acid concentrations. The basis for this correlation is the stoichiometry of the acetoacetyl-coenzyme A (CoA): acetate (butyrate) CoA transferase reaction (Hartmanis et al. 1984). For each mole of butyric acid that is converted to butyryl-CoA and subsequently reduced to butanol, one mole of acetoacetyl-CoA is converted to acetoacetate, which is subsequently decarboxylated to acetone. An exception is acid consumption without acetone production in the presence of carbon monoxide (Datta and Zeikus 1985; Meyer et al. 1986). However, this results in a number greater than one for $\Delta C_{\text {acid decrease }} / \Delta C_{\text {acetone }}$ and the oleyl alcohol extractive fermentation demonstrated a ratio of less than one. The observation that the production of acetone was correlated with the conversion of butyric acid to butanol (Table 2) established that no paradox existed.

The difference between the Type I and Type II acid concentration profiles was due to altered butyric acid production. Generally, in batch fermentation, acid production is terminated when acid conversion to alcohol begins (Jones and Woods 1986). However, the production of butyric acid was prolonged into the solventogenic phase in the oleyl alcohol extractive fermentation (Table 2). The concentration of butyric acid remained high (Fig. 1b) because butyric acid was continually being replenished from glucose while butyric acid was being converted to butanol. 
The addition of acids or CCCP did not enhance the rate of acetone production (Table 3 ). Therefore, the addition of acids or CCCP to the oleyl alcohol extractive fermentation was concluded to have not enhanced the rate of conversion of butyric acid to butanol. The observed enhancements of the rate of decrease in butyric acid concentration $(-d C / d t)$ upon the addition of acids or CCCP (Table 3 ) were attributed to decreased $r_{\text {prod }}$ and not to increased $r_{\text {conv }}$.

The uncoupler CCCP is known to decrease the $\Delta \mathrm{pH}$ in C. acetobutylicum (Huang et al. 1985). Since both acids and CCCP enhanced $-d C / d t$ (Table 3), the $\triangle \mathrm{pH}$ or the intracellular $\mathrm{pH}$ (the extracellular $\mathrm{pH}$ was constant) may have inhibited butyric acid production. Phosphotransbutyrylase (PTB), one enzyme in the pathway for butyric acid production, is sensitive to $\mathrm{pH}$ over the physiological pH range of $C$. acetobutylicum (Wiesenborn et al. 1989). A decrease in the $\Delta \mathrm{pH}$ upon the addition of butyric acid or CCCP may have led to a decrease in the intracellular $\mathrm{pH}$ and inhibited PTB.

The following model of $C$. acetobutylicum in extractive fermentation was concluded on the basis of the data presented and data in the literature. The addition of oleyl alcohol to the broth resulted in the extraction of butyric acid, which lowered the aqueous concentration of butyric acid. This lower concentration prolonged acid production, at least partially because of a greater $\Delta \mathrm{pH}$, and prevented the decline in butyric acid concentration during solventogenesis. The addition of decanol to the extractant inhibited butyric acid production and reinstated the normal acid concentration profile (Type II) possibly because decanol, like other alcohols, decreases the $\Delta \mathrm{pH}$ (Bowles and Ellefson 1985; Gottwald and Gottschalk 1985; Huang et al. 1986).

Acknowledgements. The technical and financial assistance of the Michigan Biotechnology Institute and the comments of Jean Bonnes, Kevin Dykstra, Norberto Palleroni, and Lily Young are appreciated.

\section{References}

Bahl H, Andersch W, Braun K, Gottschalk G (1982) Effect of $\mathrm{pH}$ and butyrate concentration on the production of acetone and butanol by Clostridium acetobutylicum grown in continuous culture. Eur J Appl Microbiol Biotechnol 14:17-20

Ballongue J, Amine J, Masion E, Petitdemange H, Gay R (1985) Induction of acetoacetate decarboxylase in Clostridium acetobutylicum. FEMS Microbiol Lett 29:273-277

Bowles LK, Ellefson WL (1985) Effects of butanol on Clostridium acetobutylicum. Appl Environ Microbiol 50:11651170
Datta R, Zeikus JG (1985) Modulation of acetone-butanolethanol fermentation by carbon monoxide and organic acids. Appl Environ Microbiol 49:522-529

Evans PJ, Wang HY (1988) Enhancement of butanol formation by Clostridium acetobutylicum in the presence of decanol-oleyl alcohol mixed extractants. Appl Environ Microbiol 54:1662-1667

Fond O, Matta-Ammouri G, Petitdemange H, Engasser JM (1985) The role of acids on the production of acetone and butanol by Clostridium acetobutylicum. Appl Microbiol Biotechnol 22: 195-200

Gottshal JC, Morris JG (1981) The induction of acetone and butanol production in cultures of Clostridium acefobutylicum by elevated concentrations of acetate and butyrate. FEMS Microbiol Lett 12:385-389

Gottwald M, Gottschalk G (1985) The internal pH of Clostridium acetobutylicum and its effect on the shift from acid to solvent formation. Arch Microbiol 143:42-46

Hartmanis MGN, Klason T, Gatenbeck S (1984) Uptake and activation of acetate and butyrate in Clostridium acetobutylicum. Appl Microbiol Biotechnol 20:66-71

Herbert D, Phipps PJ, Strange RE (1971) Chemical analysis of microbial cells. In: Norris JR, Ribbons DW (eds) Methods in microbiology, vol 5B, Academic Press, London, pp 209 344

Holt R, Stephens GM, Morris JG (1984) Production of solvents by Clostridium acetobutylicum cultures maintained at neutral pH. Appl Environ Microbiol 48:1166-1170

Huang L, Gibbins LN, Forsberg CW (1985) Transmembrane $\mathrm{pH}$ gradient and membrane potential in Clostridium acetobutylicum during growth under acetogenic and solventogenic conditions. Appl Environ Microbiol 50: 1043-1047

Huang L, Forsberg CW, Gibbins LN (1986) Influence of external $\mathrm{pH}$ and fermentation products on Clostridium acetobutylicum intracellular $\mathrm{pH}$ and cellular distribution of fermentation products. Appl Environ Microbiol 51:12301234

Huesemann M, Papoutsakis ET (1986) Effect of acetoacetate, butyrate and uncoupling ionophores on growth and product formation of Clostridium acetobutylicum. Biotechnol Lett $8: 37-42$

Jones DT, Woods DR (1986) Acetone-butanol fermentation revisited. Microbiol Rev 50:484-524

Meyer CL, Roos JW, Papoutsakis ET (1986) Carbon monoxide gasing leads to alcohol production and butyrate uptake without acetone formation in continuous culture of Clostridium acetobutylicum. Appl Microbiol Biotechnol 24:159-167

Roffler SR, Blanch HW, Wilke CR (1987) In-situ recovery of butanol during fermentation, part 1 : batch extractive fermentation. Bioproc Eng 2:1-12

Taya M, Ishii S, Kobayashi T (1985) Monitoring and control for extractive fermentation of Clostridium acetobutylicum. $\mathrm{J}$ Ferment Technol 63:181-187

Terracciano JS, Kashket ER (1986) Intracellular conditions required for initiation of solvent production by Clostridium acetobutylicum. Appl Environ Microbiol 52:86-91

Wiesenborn DP, Rudolph FB, Papoutsakis ET (1989) Phosphotransbutyrylase from Clostridium acetobutylicum ATCC 824 and its role in acidogenesis. Appl Environ Microbiol $55: 317-322$

Received 27 March 1989/Accepted 11 September 1989 